\title{
Nonrelativistic trace and diffeomorphism anomalies in particle number background
}

\author{
Roberto Auzzi, ${ }^{1,2}$ Stefano Baiguera, ${ }^{3}$ and Giuseppe Nardelli ${ }^{1,4}$ \\ ${ }^{1}$ Dipartimento di Matematica e Fisica, Università Cattolica del Sacro Cuore, \\ Via Musei 41, 25121 Brescia, Italy \\ ${ }^{2}$ INFN Sezione di Perugia, Via A. Pascoli, 06123 Perugia, Italy \\ ${ }^{3}$ Università degli studi di Milano Bicocca and INFN, Sezione di Milano-Bicocca, \\ Piazza della Scienza 3, 20161 Milano, Italy \\ ${ }^{4}$ TIFPA-INFN, clo Dipartimento di Fisica, Università di Trento, 38123 Povo (Trento), Italy
}

(Received 21 February 2018; published 11 April 2018)

\begin{abstract}
Using the heat kernel method, we compute nonrelativistic trace anomalies for Schrödinger theories in flat spacetime, with a generic background gauge field for the particle number symmetry, both for a free scalar and a free fermion. The result is genuinely nonrelativistic, and it has no counterpart in the relativistic case. Contrary to naive expectations, the anomaly is not gauge invariant; this is similar to the nongauge covariance of the non-Abelian relativistic anomaly. We also show that, in the same background, the gravitational anomaly for a nonrelativistic scalar vanishes.
\end{abstract}

DOI: 10.1103/PhysRevD.97.085010

\section{INTRODUCTION}

Trace anomaly gives powerful constraints on the possible degrees of freedom that can emerge in the infrared of a strongly coupled relativistic and unitary theory: the Zamolodchikov $c$ theorem in $d=2$ [1] and the $a$-theorem in $d=4$ [2-7] give us examples of monotonically decreasing quantities between the UV and the IR conformal fixed points. For condensed matter applications, it would be interesting to generalize such results to the nonrelativistic case.

Newton-Cartan (NC) geometry was originally introduced as a covariant formulation of Newtonian gravity. In recent years, it found several applications in condensed matter systems such as quantum Hall effect and fermions at unitarity; see, e.g., [8-11]. For theories with Schrödinger invariance, $\mathrm{NC}$ gravity provides a natural set of sources for the operators in the energy-momentum tensor multiplet.

Promising candidates for nonrelativistic $a$-theorems are given by type-A trace anomalies [12]. In the case of Schrödinger-invariant theories coupled to NC geometry in $2+1$ dimensions, a natural candidate for a monotonically decreasing $a$-function was introduced in [13] and further studied in [14-20]. All these works (with the exception of [19]) assume that the trace anomaly is invariant under diffeomorphisms, Milne and $U(1)$ gauge transformations. The case of Lifshitz theories was studied, e.g., in [21-27], and no natural

Published by the American Physical Society under the terms of the Creative Commons Attribution 4.0 International license. Further distribution of this work must maintain attribution to the author(s) and the published article's title, journal citation, and DOI. Funded by SCOAP . candidate for a monotonically decreasing type-A term in the trace anomaly was found so far.

In general, anomalies correspond to violations of current conservation in the presence of background fields. In the simplest incarnations, such as the ABJ chiral anomaly $[28,29]$, they can be written as gauge-invariant functions. Subsequently, Bardeen [30] showed that anomalies may not be gauge covariant: gauge invariance for background fields might be formally lost in the regularization procedure. Such gaugeviolating terms are not just a feature of chiral anomalies; they can appear also in trace anomalies [31]. As discussed in [32], the presence of these terms in supersymmetric theories is instrumental in deriving a-maximization [33] using Osborn's local renormalization group formalism [3-5].

The first explicit calculation of the Schrödinger trace anomaly in NC background was performed in [17] for the scalar case and in [18] for the fermionic case. There, the heat kernel procedure was used and a NC background with vanishing particle number gauge potential was chosen. With this assumption, the trace anomaly result was gauge and Milneboost invariant. Subsequently, Ref. [19] did a related calculation in the scalar case using the Fujikawa method with a NC background with a nonvanishing $U(1)$ particle number. ${ }^{1}$ gauge field. Surprisingly, the trace anomaly was not $U(1)$ gauge invariant.

\footnotetext{
${ }^{1}$ More correctly, this $U(1)$ symmetry in the presence of different species of fields $\psi_{i}$ corresponds to the mass, because in the minimal coupling it enters the action as $-\sum_{i} m_{i} A_{0}\left|\psi_{i}\right|^{2}$, where $m_{i}$ is the mass of the field $\psi_{i}$. In the presence of a single species, mass and particle number are proportional to each other. For simplicity, we refer to this $U(1)$ symmetry as particle number.
} 
In this paper, we investigate such violations with the heat kernel formalism. We consider the cases of a free nonrelativistic scalar and a free fermion in $2+1$ dimensions, and we compute the expectation value of the trace of the energy-momentum tensor for a flat background geometry and a generic source $A_{\mu}$ for the particle number.

Moreover, Ref. [19] also found a nonvanishing diffeomorphism anomaly, both in the presence of spacetime curvature and a $U(1)$ gauge field. Given the recent interesting applications of diffeomorphism anomaly in condensed matter systems (see, e.g., [34-36]), one of the motivations for this work is also to deepen and understand the nature of this anomaly in nonrelativistic theories. We compute this anomaly in the presence of a background gauge field and, surprisingly, we find a vanishing result. As we shall explain, this is not necessarily in contradiction to [19]: we find that it is possible to define a "subtracted" energy-momentum tensor which is conserved, in the spirit of [37]. Therefore, the apparent difference may be all due to a different renormalization and subtraction procedure.

This paper is organized as follows: in Sec. II, we introduce the notation and the sources for the background currents. In Sec. III, we compute the trace anomaly in a particle number background both for a nonrelativistic boson and a fermion. In Sec. IV, we discuss diffeomorphism anomaly for a nonrelativistic scalar and we compare the results with [19]. We conclude in Sec. V, and technical details are deferred to the Appendixes.

\section{PRELIMINARIES AND NOTATION}

We will consider a nonrelativistic free scalar and fermion in $2+1$ dimensions coupled to a NC background geometry. As a useful method to deal in a convenient way with all the spacetime symmetries, we will use the null-reduction trick [38] from an extra-dimensional relativistic $3+$ 1-dimensional theory. Useful references about NC geometry formalism include [39-46].

Since we are dealing with fermions, we will need both curved spacetime indices and tangent space ones for the frame field. The index conventions that we shall use are as follows: late latin capital indices (e.g., $M, N, \ldots$ ), denote $3+1$-dimensional curved spacetime indices; early latin capital indices (e.g., $A, B, \ldots$,) denote tangent space indices, whose metric is locally flat. The coordinate $x^{-}$ corresponds to the null-reduction direction. The extradimensional indices of the curved space and of the locally flat tangent space are as follows:

$$
\begin{aligned}
M & =(-, \mu)=(-,+, i) \quad(i=1,2) \\
A & =(-, \alpha)=(-,+, a) \quad(a=1,2),
\end{aligned}
$$

where + denotes the nonrelativistic time direction and $i, a$ the space ones (for the curved, tangent space case, respectively).
The NC spacetime geometry is described by a positive definite symmetric rank-two tensor $h^{\mu \nu}$ (which corresponds to the spatial inverse metric) and by a nowhere vanishing vector $n_{\mu}$ (defining the local time direction), with the hortogonality condition

$$
n_{\mu} h^{\mu \nu}=0
$$

A velocity field $v^{\mu}$ is also introduced, with the condition

$$
n_{\mu} v^{\mu}=1
$$

Given $\left(h^{\mu \nu}, n_{\mu}, v^{\nu}\right)$, one can then uniquely define the spatial metric $h_{\mu \nu}$, with

$$
h^{\mu \rho} h_{\rho \nu}=\delta_{\nu}^{\mu}-v^{\mu} n_{\nu} \equiv P_{\nu}^{\mu}, \quad h_{\mu \alpha} v^{\alpha}=0,
$$

where $P_{\nu}^{\mu}$ is the projector onto spatial directions. We introduce also a nondynamical gauge field $A_{\mu}$ as a source for the particle number symmetry. ${ }^{2}$ In terms of the $2+$ 1-dimensional quantities, the extra-dimensional metric used in the null reduction is

$$
\begin{aligned}
G_{M N} & =\left(\begin{array}{cc}
0 & n_{\nu} \\
n_{\mu} & n_{\mu} A_{\nu}+n_{\nu} A_{\mu}+h_{\mu \nu}
\end{array}\right), \\
G^{M N} & =\left(\begin{array}{cc}
A^{2}-2 v \cdot A & v^{\nu}-h^{\nu \sigma} A_{\sigma} \\
v^{\mu}-h^{\mu \sigma} A_{\sigma} & h^{\mu \nu}
\end{array}\right),
\end{aligned}
$$

where $A^{2}-2 v \cdot A=h^{\mu \nu} A_{\mu} A_{\nu}-2 v^{\mu} A_{\mu}$. The determinant of the metric is then

$$
\sqrt{g}=\sqrt{-\operatorname{det} G_{A B}}=\sqrt{\operatorname{det}\left(h_{\mu \nu}+n_{\mu} n_{\nu}\right)} .
$$

The symmetries of the Newton-Cartan theory include, besides diffeomorphisms and local U(1) gauge invariance, a local version of Galilean boosts, namely the Milne boosts. Transformation properties of fields under Weyl and Milne boosts are reported in Appendix A.

To deal with spinors, it is necessary to introduce an orthonormal frame field which relates the metric in the curved spacetime with the flat tangent space. The flat tangent space metric is

$$
G_{A B}=G^{A B}=\left(\begin{array}{cccc}
0 & 1 & 0 & 0 \\
1 & 0 & 0 & 0 \\
0 & 0 & 1 & 0 \\
0 & 0 & 0 & 1
\end{array}\right),
$$

and the frame fields are defined by

\footnotetext{
${ }^{2}$ The presence of the vector field is related to the arbitrariness $v^{\mu} \rightarrow v^{\mu}+h^{\mu \nu} A_{\nu}$ one has in defining the velocity field.
} 


$$
\begin{aligned}
G_{M N} & =e^{A}{ }_{M} G_{A B} e^{B}{ }_{N}, \quad G_{A B}=e^{M}{ }_{A} G_{M N} e^{N}{ }_{B}, \\
e^{A}{ }_{M} e^{M}{ }_{B} & =\delta^{A}{ }_{B}, \quad e^{M}{ }_{A} e^{A}{ }_{N}=\delta^{M}{ }_{N} .
\end{aligned}
$$

The spin connection associated to the vielbein is

$$
\begin{aligned}
\omega_{M A B}= & \frac{1}{2}\left[e^{N}{ }_{A}\left(\partial_{M} e_{N B}-\partial_{N} e_{M B}\right)-e^{N}{ }_{B}\left(\partial_{M} e_{N A}-\partial_{N} e_{M A}\right)\right. \\
& \left.-e^{N}{ }_{A} e^{P}{ }_{B}\left(\partial_{N} e_{P C}-\partial_{P} e_{N C}\right) e^{C}{ }_{M}\right] .
\end{aligned}
$$

The explicit form of the fierbein, dreibein, and their inverses as well as conventions on gamma matrices are summarized in Appendix B.

\section{A. Sources and conserved currents}

Let us consider a generic nonrelativistic matter field $\phi$ coupled to a NC background (collectively denoted by $g$ ). The vacuum functional $W[g]$ is defined as the quantum average of the action over the matter fields, namely as the path integral:

$$
e^{i W[g]}=\int \mathcal{D} \phi^{*} \mathcal{D} \phi e^{i S\left[\phi, \phi^{*}, g\right]}
$$

The NC fields act as sources in the vacuum functional, so that it is possible to generate all the Ward identities by functional differentiation of $W[g]$ with respect to the NC sources. However, due to the constraints (2.2)-(2.4) relating the $\mathrm{NC}$ entrees, arbitrary variations on background fields are not allowed, and one must first identify the independent variations, e.g., [11]. These can be parametrized in terms of an arbitrary $\delta n_{\mu}$, a transverse perturbation $\delta u^{\mu}$ with $\delta u^{\mu} n_{\mu}=0$, and a transverse metric perturbation $\delta \tilde{h}^{\alpha \beta} n_{\beta}=0$. Then, the variation of the NC metric in terms of the independent variations can be written as

$$
\begin{aligned}
\delta n_{\mu}, \quad \delta v^{\mu} & =-v^{\mu} v^{\alpha} \delta n_{\alpha}+\delta u^{\mu}, \\
\delta h^{\mu \nu} & =-v^{\mu} \delta n^{\nu}-\delta n^{\mu} v^{\nu}-\delta \tilde{h}^{\mu \nu} .
\end{aligned}
$$

Consequently, the NC metric nearby the flat limit (2.7) gives

$$
\begin{aligned}
n_{\mu} & =\left(1+\delta n_{0}, \delta n_{i}\right), \quad v^{\mu}=\left(1-\delta n_{0}, \delta u_{i}\right), \\
\delta \tilde{h}^{0 i} & =0, h_{\mu \nu}=\left(\begin{array}{cc}
0 & -\delta u_{i} \\
-\delta u_{i} & \delta_{i j}+\delta \tilde{h}_{i j}
\end{array}\right), \\
h^{\mu \nu} & =\left(\begin{array}{cc}
0 & -\delta n_{i} \\
-\delta n_{i} & \delta_{i j}-\delta \tilde{h}_{i j}
\end{array}\right) .
\end{aligned}
$$

The null reduction metric is

$$
\begin{aligned}
G_{A B} & =\left(\begin{array}{ccc}
0 & 1+\delta n_{0} & \delta n_{i} \\
1+\delta n_{0} & 2 \delta A_{0} & \delta A_{i}-\delta u_{i} \\
\delta n_{i} & \delta A_{i}-\delta u_{i} & \delta_{i j}+\delta \tilde{h}_{i j}
\end{array}\right), \\
G^{A B} & =\left(\begin{array}{ccc}
-2 A_{0} & 1-\delta n_{0} & -\delta A_{i}+\delta u_{i} \\
1-\delta n_{0} & 0 & -\delta n_{i} \\
-\delta A_{i}+\delta u_{i} & -\delta n_{i} & \delta_{i j}-\delta \tilde{h}_{i j}
\end{array}\right) .
\end{aligned}
$$

We can use these sources to define the currents of the energy-momentum tensor multiplets: under the above infinitesimal variations, the vacuum functional varies according to

$$
\delta W=\int d^{d} x \sqrt{-g}\left(\frac{1}{2} T_{i j} \delta \tilde{h}_{i j}+j^{\mu} \delta A_{\mu}-\epsilon^{\mu} \delta n_{\mu}-p_{i} \delta u_{i}\right) .
$$

Here, $p_{i}$ is the momentum density, $T_{i j}$ is the spatial stress tensor, $j^{\mu}=\left(j^{0}, j^{i}\right)$ contains the $U(1)$ number density and current, and $\epsilon^{\mu}=\left(\epsilon^{0}, \epsilon^{i}\right)$ is the energy density and current. Here, $\epsilon^{\mu}$ includes also the contribution coming from the "chemical potential" $A_{0}$; see, e.g., Eq. (3.2). Note that in Eq. (2.13), the variations $\delta A_{i}$ and $\delta u_{i}$ do not appear independently but always in the combination $\delta A_{i}-\delta u_{i}$. As a consequence of this fact, the $U(1)$ number particle current is always proportional to the momentum density, as it should be in a nonrelativistic theory.

Ward identities in the flat limit can be easily obtained in the usual way: consider a symmetry of the classical action, specify the corresponding infinitesimal variation of the NC metric, and impose invariance of the functional, $\delta W=0$. Then, associated with particle number conservation, there is conservation of the $U(1)$ current:

$$
\left\langle\partial_{\mu} j^{\mu}\right\rangle=0 .
$$

Associated with diffeomorphism invariance, there are the conservation of the spatial stress tensor and the energy current conservation:

$$
\left\langle\partial_{t} p^{j}+\partial_{i} T^{i j}\right\rangle=0, \quad\left\langle\partial_{\mu} \epsilon^{\mu}\right\rangle=0 .
$$

Finally, local Weyl transformation entails the Ward identity associated with the conservation of the scale current, which is found to $\mathrm{be}^{3}$

$$
J_{S}^{0}=p_{i} x^{i}-2 t \epsilon^{0}, \quad J_{S}^{i}=x^{j} T^{i}{ }_{j}-2 t \epsilon^{i}, \quad\left\langle\partial_{\mu} J_{S}^{\mu}\right\rangle=0 .
$$

\footnotetext{
${ }^{3}$ Strictly speaking, the scale current has an additional term proportional to the scaling dimension $\Delta$ of the matter field. However, such a term is a total derivative and can always be reabsorbed by a current redefinition.
} 
By expanding explicitly the scale Ward identity, we have $\left\langle\partial_{\mu} J_{S}^{\mu}\right\rangle=\left\langle T^{i}{ }_{i}-2 \epsilon^{0}\right\rangle-2 t\left\langle\partial_{\mu} \epsilon^{\mu}\right\rangle+x^{j}\left\langle\partial_{t} p_{j}+\partial_{i} T^{i}{ }_{j}\right\rangle=0$.

Equation (2.18) is interesting, as it reveals the relations intertwining between tracelessness of the energy-momentum tensor, conservation of the energy momentum tensor, and scale conservation. A quantum violation of the scale symmetry manifests as a nonconservation of the scale current $J_{S}^{\mu}$ which, in turn, is equivalent to a violation of the tracelessness condition $\left\langle T^{i}{ }_{i}-2 \epsilon^{0}\right\rangle=0$ only if the energymomentum tensor does not have a diffeomorphism anomaly, i.e., only if the conditions (2.16) are satisfied. On the contrary, if the energy-momentum tensor is not conserved at the quantum level, not only the trace anomaly but also the diffeomorphism anomaly contribute to the scale anomaly.

\section{B. Flat spacetime with $U(1)$ gauge field}

We will compute the trace anomaly for a flat background where only the nondynamical $U(1)$ gauge potential is switched on,

$n_{\mu}=(1, \mathbf{0}), \quad v^{\mu}=(1, \mathbf{0}), \quad h_{i j}=\delta_{i j}$,

$A_{\mu}=\left(A_{0}\left(t, x^{i}\right), A_{i}\left(t, x^{i}\right)\right)$,

which corresponds to the extra-dimensional metric

$$
\begin{aligned}
G_{M N} & =\left(\begin{array}{cccc}
0 & 1 & 0 & 0 \\
1 & 2 A_{0} & A_{1} & A_{2} \\
0 & A_{1} & 1 & 0 \\
0 & A_{2} & 0 & 1
\end{array}\right), \\
G^{M N} & =\left(\begin{array}{cccc}
-2 A_{0}+A_{i} A_{i} & 1 & -A_{1} & -A_{2} \\
1 & 0 & 0 & 0 \\
-A_{1} & 0 & 1 & 0 \\
-A_{2} & 0 & 0 & 1
\end{array}\right) .
\end{aligned}
$$

The vielbein is

$$
\begin{aligned}
e^{A}{ }_{M} & =\left(\begin{array}{cccc}
1 & A_{0} & A_{1} & A_{2} \\
0 & 1 & 0 & 0 \\
0 & 0 & 1 & 0 \\
0 & 0 & 0 & 1
\end{array}\right), \\
e^{M}{ }_{A} & =\left(\begin{array}{cccc}
1 & -A_{0} & -A_{1} & -A_{2} \\
0 & 1 & 0 & 0 \\
0 & 0 & 1 & 0 \\
0 & 0 & 0 & 1
\end{array}\right) .
\end{aligned}
$$

Starting from these data, we can compute the nonvanishing components of the spin connection
$\omega_{++i}=-F_{0 i}, \quad \omega_{+i j}=-\frac{1}{2} F_{i j}, \quad \omega_{i+j}=-\frac{1}{2} F_{i j}$,

and the nonvanishing components of the Christoffel symbol $^{4}$ :

$\Gamma_{\mu \nu}^{-}=\frac{1}{2}\left(v_{A}\right)^{\sigma}\left(Q_{A}\right)_{\mu \nu \sigma}, \quad \Gamma_{\mu \nu}^{\rho}=\frac{1}{2} h^{\rho \sigma}\left(Q_{A}\right)_{\mu \nu \sigma}$,

where

$$
\begin{aligned}
F_{\mu \nu} & =\partial_{\mu} A_{\nu}-\partial_{\nu} A_{\mu}, \\
\left(v_{A}\right)^{\mu} & =v^{\mu}-h^{\mu \nu} A_{\nu}, \\
\left(h_{A}\right)_{\mu \nu} & =h_{\mu \nu}+A_{\mu} n_{\nu}+A_{\nu} n_{\mu}, \\
\left(Q_{A}\right)_{\mu \nu \sigma} & =\partial_{\mu}\left(h_{A}\right)_{\nu \sigma}+\partial_{\nu}\left(h_{A}\right)_{\mu \sigma}-\partial_{\sigma}\left(h_{A}\right)_{\mu \nu} .
\end{aligned}
$$

\section{TRACE ANOMALY}

The action for a nonrelativistic boson is

$$
\begin{aligned}
S= & \int d^{3} x \sqrt{g}\left[i m v^{\mu} \phi^{\dagger} D_{\mu} \phi-i m v^{\mu}\left(D_{\mu} \phi\right)^{\dagger} \phi\right. \\
& \left.-h^{\mu \nu}\left(D_{\mu} \phi\right)^{\dagger} D_{\nu} \phi-\xi R \phi^{\dagger} \phi\right],
\end{aligned}
$$

where $D_{\mu}=\partial_{\mu}-i m A_{\mu}$. Specializing to a flat background and performing an integration by parts, we find

$$
\begin{aligned}
S= & \int d^{3} x\left[2 i m \phi^{\dagger} \partial_{t} \phi+\phi^{\dagger} \partial_{i}^{2} \phi-2 i m A_{i} \phi^{\dagger} \partial_{i} \phi\right. \\
& \left.+\left(2 m^{2} A_{0}-m^{2} A_{i} A_{i}-i m \partial_{i} A_{i}\right) \phi^{\dagger} \phi\right] .
\end{aligned}
$$

Note that $A_{0}$ plays the role of a grand canonical chemical potential coupled to the particle number $J_{0}=2 m^{2} \phi^{\dagger} \phi$. As a consequence, $\epsilon^{0}=E+A_{0} J^{0}$, where $E$ is the particle energy density.

\section{A. The unperturbed case}

We shall compute the trace anomaly using the heat kernel (HK) method in imaginary time space. The following substitutions are used [47]

$$
t \rightarrow-i t_{E}, \quad \partial_{t} \rightarrow i \partial_{t_{E}}, \quad m \rightarrow i m_{E} .
$$

For a generic operator $\hat{\mathcal{O}}_{E}$, the $\mathrm{HK}$ operator of $\hat{\mathcal{O}}_{E}$ is defined as

$$
\hat{K}_{\hat{\mathcal{O}}_{E}}(s)=\exp \left(s \hat{\mathcal{O}}_{E}\right)
$$

\footnotetext{
${ }^{4}$ In the following, the presence of the subscript $A$ denotes that the corresponding quantity is Milne-boost invariant
} 
The following matrix elements are introduced,

$$
K_{\hat{\mathcal{O}}_{E}}\left(s, x, t, x^{\prime}, t^{\prime}\right)=\left\langle x t\left|\hat{K}_{\hat{\mathcal{O}}_{E}}(s)\right| x^{\prime} t^{\prime}\right\rangle,
$$

and we denote by $\tilde{K}_{\hat{\mathcal{O}}_{E}}$ the diagonal matrix elements,

$$
\tilde{K}_{\hat{\mathcal{O}}_{E}}(s, x, t)=\left\langle x t\left|\hat{K}_{\hat{\mathcal{O}}_{E}}(s)\right| x t\right\rangle .
$$

The HK is an efficient method to compute the one-loop effective action; in the unpertubed flat nonrelativistic case with $A_{\mu}=0$, the free Schrödinger operator $\triangle$ entering the action is given by

$$
\triangle=\left(-2 i m \partial_{t}+\partial_{i}^{2}\right)=\left(-2 m \sqrt{-\partial_{t}^{2}}+\partial_{i}^{2}\right) .
$$

The HK matrix elements $K_{\triangle}$ have been computed in [17],

$$
\begin{aligned}
K_{\triangle}(s) & =\left\langle x t\left|e^{s \Delta}\right| x^{\prime} t^{\prime}\right\rangle \\
& =\frac{1}{2 \pi} \frac{m s}{m^{2} s^{2}+\frac{\left(t-t^{\prime}\right)^{2}}{4}} \frac{1}{(4 \pi s)} \exp \left(-\frac{\left(x-x^{\prime}\right)^{2}}{4 s}\right) .
\end{aligned}
$$

\section{B. The perturbative expansion}

In curved space one introduces the following scalar product in coordinate representation:

$$
\left\langle x t \mid x^{\prime} t^{\prime}\right\rangle_{g}=\frac{\delta\left(x-x^{\prime}\right) \delta\left(t-t^{\prime}\right)}{\sqrt{g}} .
$$

In a generic background, it is convenient to expand the complete Schrödinger operator $\hat{\triangle}$ as the sum of its free part $\triangle$ plus a perturbation $\hat{V}$. We can then evaluate the HK as a perturbative expansion around (3.8). The diagonal elements of the HK operator can be expanded in powers of $s$ as

$$
\begin{aligned}
\tilde{K}_{\hat{\triangle}}(s) & =\operatorname{Tr}\left\langle x t\left|e^{s \hat{\Delta}}\right| x t\right\rangle_{g} \\
& =\frac{1}{s^{2}}\left(a_{0}(\hat{\triangle})+a_{2}(\hat{\triangle}) s+a_{4}(\hat{\triangle}) s^{2}+\cdots\right) .
\end{aligned}
$$

This expansion provides the definition of the De WittSeeley-Gilkey coefficients $a_{2 k}(\hat{\triangle})$. For a nonrelativistic $2+1$-dimensional theory, the trace anomaly is proportional to the $a_{4}$ coefficient [17].

In general, it is convenient to work in a quantum mechanical space with flat inner product

$$
\left\langle x t \mid x^{\prime} t^{\prime}\right\rangle=\delta\left(x-x^{\prime}\right) \delta\left(t-t^{\prime}\right) .
$$

Consequently, for any operator $\hat{\mathcal{O}}$ we can define the operator $\hat{M}_{\hat{\mathcal{O}}}$ such that

$$
\left\langle x t|\hat{\mathcal{O}}| x^{\prime} t^{\prime}\right\rangle_{g}=\left\langle x t\left|\hat{M}_{\hat{\mathcal{O}}}\right| x^{\prime} t^{\prime}\right\rangle
$$

The "effective" operator $\hat{M}_{\hat{\mathcal{O}}}$ keeps track of the metric in the inner product. In this way we can expand the diagonal elements of the HK as

$$
\begin{aligned}
\tilde{K}_{\hat{M}}(s) & =\operatorname{Tr}\left\langle x t\left|e^{s \hat{M}}\right| x t\right\rangle \\
& =\frac{1}{s^{2}}\left[a_{0}(\hat{M})+s a_{2}(\hat{M})+s^{2} a_{4}(\hat{M})+\cdots\right] \\
& \equiv \sqrt{g} \tilde{K}_{\hat{\Delta}}(s) .
\end{aligned}
$$

In our flat case, $\sqrt{g}=1$ and so $\hat{\mathcal{O}}=\hat{M}_{\hat{\mathcal{O}}}$.

We can parametrize the perturbation from the flat contribution as

$$
\begin{aligned}
\left\langle x t|\hat{M}| x^{\prime} t^{\prime}\right\rangle= & \left\langle x t|\triangle+\hat{V}| x^{\prime} t^{\prime}\right\rangle \\
= & \langle x t| \triangle+P(x) \delta\left(x-x^{\prime}\right) \delta\left(t-t^{\prime}\right) \\
& +S(x) \sqrt{-\partial_{t}^{2}} \delta\left(x-x^{\prime}\right) \delta\left(t-t^{\prime}\right) \\
& +Q_{i}(x) \partial_{i} \delta\left(x-x^{\prime}\right) \delta\left(t-t^{\prime}\right)\left|x^{\prime} t^{\prime}\right\rangle .
\end{aligned}
$$

The perturbative calculation starts by considering the expansion

$$
K_{\hat{M}}(s)=\exp (s(\triangle+\hat{V}))=\sum_{n=0}^{\infty} K_{n}(s),
$$

where the single terms entering the series are obtained via a Dyson recursive procedure:

$$
\begin{aligned}
K_{n}(s)= & \int_{0}^{s} d s_{n} \int_{0}^{s_{n}} d s_{n-1} \ldots \\
& \times \int_{0}^{s_{2}} d s_{1} e^{\left(s-s_{n}\right) \triangle} \hat{V} \ldots e^{\left(s_{2}-s_{1}\right) \triangle} \hat{V} e^{s_{1} \triangle} .
\end{aligned}
$$

The terms $K_{n}(s)$ consists of insertions of $n$ operators among the set

$$
\left\{P(x), S(x), Q_{i}(x)\right\}
$$

and then the computation is performed when we find the value of $K_{n}(s)$ for all the nonvanishing combinations of terms in the previous set.

The imaginary time rotation of the gauge field gives ${ }^{5}$ :

$$
A_{0} \rightarrow A_{0} \quad A_{i} \rightarrow-i A_{i}
$$

and the imaginary time action reads:

\footnotetext{
${ }^{5}$ The unconventional redefinition of the gauge field in the imaginary time formalism is required by consistency with $\left[D_{\mu}, D_{\nu}\right]=-i m F_{\mu \nu}$ and the prescription $m \rightarrow i m$. The imaginary mass is required in order to get a positive definite euclidean action.
} 


$$
\begin{aligned}
S_{E}= & -\int d^{3} x \phi^{\dagger}\left[\triangle-2 i m A_{i} \partial_{i}-2 m^{2} A_{0}\right. \\
& \left.-m^{2} A_{i} A_{i}-i m\left(\partial_{i} A_{i}\right)\right] \phi .
\end{aligned}
$$

We can immediately identify

$$
\begin{aligned}
S\left(t, x^{i}\right) & =0, \quad P\left(t, x^{i}\right)=-2 m^{2} A_{0}-m^{2} A_{i} A_{i}-i m\left(\partial_{i} A_{i}\right), \\
Q_{i}\left(t, x^{i}\right) & =-2 i m A_{i},
\end{aligned}
$$

so that all the insertions containing at least one operator $S\left(t, x^{i}\right)$ vanish. Therefore, at the first order $(n=1)$, there are just two terms, denoted by $K_{1 P}$ and $K_{1 Q_{i}}$. At the second order (double insertion, $n=2$ ) we have four possible insertions, $K_{2 P P}, K_{2 P Q_{j}}, K_{2 Q_{i} P}, K_{2 Q_{i} Q_{j}}$.

For time independent backgrounds, the calculation of the coefficients can be found in [17-18] with the exception of the $K_{1 Q_{i}}$ term, which was not needed neither for the scalar nor for the fermion anomaly. Here in Appendix $\mathrm{C}$ we calculate such a term. In Appendix D, instead, we provide all the generalizations needed for all the terms when the background is time dependent. For the single insertion, we get

$$
\begin{gathered}
\tilde{K}_{1 P}=\frac{1}{8 m \pi^{2} s^{2}} \operatorname{Tr}\left(s P+\frac{1}{6} s^{2} \partial_{x}^{2} P+\cdots\right), \\
\tilde{K}_{1 Q_{i}}=\frac{1}{8 m \pi^{2} s^{2}} \operatorname{Tr}\left(-\frac{s}{2} \partial_{i} Q_{i}-\frac{s^{2}}{12} \partial_{i} \partial^{2} Q_{i}+\cdots\right),
\end{gathered}
$$

where $\operatorname{Tr}$ denotes a trace over indices such as internal or spinorial ones (in the scalar case the trace is redundant). Substituting Eq. (3.20) in (3.21) and (3.22), and matching the powers of $s$ in Eq. (3.13), one can get the contribution to the $a_{2}$ and $a_{4}$ coefficients coming from the single insertion.

Concerning the contribution coming from the double insertion, from Appendix D one gets the general formulae

$$
\begin{gathered}
\tilde{K}_{2 P P}=\frac{1}{8 m \pi^{2} s^{2}} \operatorname{Tr}\left(\frac{s^{2}}{2} P(x)^{2}+\cdots\right), \\
\tilde{K}_{2 Q_{j} Q_{i}}=\frac{1}{8 m \pi^{2} s^{2}} \operatorname{Tr}\left[-\frac{s}{4} Q_{i} Q_{i}-\frac{s^{2}}{12} Q_{i}\left(\partial_{i} \partial_{j} Q_{j}\right)+\frac{s^{2}}{12}\left(\partial_{i} \partial_{j} Q_{i}\right) Q_{j}-\frac{s^{2}}{24}\left(\partial_{j} Q_{i}\right)\left(\partial_{i} Q_{j}\right)\right. \\
\left.+\frac{s^{2}}{8}\left(\partial_{i} Q_{i}\right)\left(\partial_{j} Q_{j}\right)-\frac{s^{2}}{12} Q_{i}\left(\partial^{2} Q_{i}\right)-\frac{s^{2}}{24}\left(\partial_{i} Q_{j}\right)^{2}+\cdots\right], \\
\tilde{K}_{2 Q_{i} P}=\frac{1}{8 m \pi^{2} s^{2}} \operatorname{Tr}\left(-\frac{s^{2}}{3} P\left(\partial_{i} Q_{i}\right)-\frac{s^{2}}{6}\left(\partial_{i} P\right) Q_{i}+\cdots\right), \\
\tilde{K}_{2 P Q_{i}}=\frac{1}{8 m \pi^{2} s^{2}} \operatorname{Tr}\left(\frac{s^{2}}{6} Q_{i}\left(\partial_{i} P\right)-\frac{s^{2}}{6}\left(\partial_{i} Q_{i}\right) P+\cdots\right) .
\end{gathered}
$$

With the above formulas, in a similar way, one gets the contributions to $a_{2}$ and $a_{4}$ coming from the double insertions. Summing all together, and extracting the null power of $s$ in (3.13), we easily get the $a_{4}$ coefficient up to the second order in the fields, and therefore the trace anomaly

$$
a_{4}=\left\langle T^{i}{ }_{i}-2 \epsilon^{0}\right\rangle=-\frac{m}{8 \pi^{2}}\left(\frac{1}{3} \partial^{2} A_{0}+\frac{1}{6} B^{2}-2 m^{2} A_{0}^{2}+\mathcal{O}\left(A_{\mu}^{3}\right)\right) \equiv \mathcal{A},
$$

where $B=F_{12}$. Equation (3.27) deserves few comments. First of all, the anomaly breaks both Milne boost and gauge invariance. Due to the intimate relationship intertwining the two symmetries, ${ }^{6}$ it is not surprising that breaking one of them does entail the breaking of the other. In addition, note that in (3.27) the $\partial^{2} A_{0}$ term does not serve to rebuild a divergence of the electric field, as it would be if the result were gauge invariant. Rather, in (3.27), $A_{0}$ should be

\footnotetext{
${ }^{6}$ In the Bargmann algebra, the commutator of the momentum and a boost is the particle number generator.
}

considered as $v^{\mu} A_{\mu}$, otherwise the first two terms would not have the correct Weyl weight (see Appendix A).

Concerning the $\partial^{2}\left(v^{\mu} A_{\mu}\right)$ term in Eq. (3.27), it can be reabsorbed by a local counterterm in the vacuum functional $W$ proportional to $R v^{\mu} A_{\mu}$. This is not possible for the $\left(v^{\mu} A_{\mu}\right)^{2}$ term, that is therefore a (type-B) genuine anomaly.

Both in the free scalar and free fermion examples, the field $A_{0}$ plays the role of an external chemical potential for the particle number $J_{0}$; in the multiple species case, $J_{0}$ plays the role of mass density. Moreover, studying geodesics in a NC background, one sees that $A_{0}$ can also be 
identified as the Newtonian gravitational potential. On physical ground one would expect mass conservation in an external gravitational field. On the other hand, the breaking of gauge invariance in Eq. (3.27) may hint a violation of the conservation of the $U(1)$ current; if this would be the case, this would be puzzling because it would not be consistent with the physical intuition. This point is beyond the purpose of the present paper and deserves further investigation.

\section{The fermion}

The Dirac operator is expressed as

$$
D=\gamma^{M} D_{M}=\gamma^{A} e^{M}{ }_{A} D_{M},
$$

Conventions on gamma matrices with lightcone indices are summarized in Appendix B and are the same used in [18]. The covariant derivative acting on fermions is

$$
\begin{aligned}
D_{M} \Psi & =\left(\partial_{M}+\frac{1}{4} \omega_{M A B} \gamma^{A B}\right) \Psi \\
& =\left(\partial_{M}+\frac{1}{8} \omega_{M A B}\left[\gamma^{A}, \gamma^{B}\right]\right) \Psi,
\end{aligned}
$$

$\omega_{M A B}$ being the spin connection. We can write the nonrelativistic fermion action in 2+1-dimensions from the null reduction of the $3+1$-dimensional Dirac action:

$$
S=\int d^{4} x \sqrt{g} i \bar{\Psi} \not D \Psi
$$

using the following profile for the fermion along the extra dimension:

$$
\Psi\left(x^{M}\right)=\psi\left(x^{\mu}\right) e^{i m x^{-}} .
$$

In the fermionic case, the imaginary-time Dirac operator $D$ is not elliptic. In order to avoid this difficulty, the squared Dirac operator $D^{2}$ is used to compute the vacuum functional:

$$
i W=\frac{1}{2} \log \operatorname{det}\left(D^{2}\right) .
$$

This trick is used both in the relativistic, see, e.g., [48], and nonrelativistic case [18]. Specializing to a flat background geometry, i.e., $\sqrt{g}=1, R=0$, and going to imaginary time, we find:

$$
\begin{aligned}
D_{E}^{2} \Psi= & \triangle \Psi-2 m^{2} A_{0} \Psi-m^{2} A_{k} A_{k} \Psi-i m\left(\partial_{i} A_{i}\right) \Psi-2 i m A_{i}\left(\partial_{i} \Psi\right)+ \\
& -m F_{i 0} \gamma^{+i} \Psi-\frac{1}{4} i m F_{i j} \gamma^{i j} \Psi+\frac{1}{2} m A_{i} F_{i j} \gamma^{+j} \Psi+\frac{1}{2} i F_{i j} \gamma^{+j}\left(\partial_{i} \Psi\right)+\frac{1}{4} i\left(\partial_{i} F_{i j}\right) \gamma^{+j} \Psi .
\end{aligned}
$$

According to Eq. (3.14), we can identify

$$
\begin{gathered}
S\left(t, x^{i}\right)=0, \quad Q_{i}\left(t, x^{i}\right)=\left(-2 i m A_{i}\right) \mathbf{1}+\frac{1}{2} i F_{i j} \gamma^{+j}, \\
P\left(t, x^{i}\right)=\left[-2 m^{2} A_{0}-m^{2} A_{k} A_{k}-i m\left(\partial_{i} A_{i}\right)\right] \mathbf{1} \\
-m F_{i 0} \gamma^{+i}-\frac{1}{4} i m F_{i j} \gamma^{i j}+\frac{1}{2} m A_{i} F_{i j} \gamma^{+j}+\frac{1}{4} i\left(\partial_{i} F_{i j}\right) \gamma^{+j},
\end{gathered}
$$

where the Dirac matrices read:

$$
\gamma^{+1}=\left(\begin{array}{cccc}
0 & \sqrt{2} & 0 & 0 \\
0 & 0 & 0 & 0 \\
0 & 0 & 0 & 0 \\
0 & 0 & -\sqrt{2} & 0
\end{array}\right), \quad \gamma^{+2}=\left(\begin{array}{cccc}
0 & -\sqrt{2} i & 0 & 0 \\
0 & 0 & 0 & 0 \\
0 & 0 & 0 & 0 \\
0 & 0 & -\sqrt{2} i & 0
\end{array}\right), \quad \gamma^{12}=\left(\begin{array}{cccc}
-i & 0 & 0 & 0 \\
0 & i & 0 & 0 \\
0 & 0 & -i & 0 \\
0 & 0 & 0 & i
\end{array}\right) .
$$

We can now use Eqs. (3.21)-(3.22) and Eqs. (3.23)-(3.26) to evaluate the single and double insertion contributions. Summing both the first and the second order terms in the external background fields, we find

$$
a_{4}\left(D_{E}^{2}\right)=-\frac{m}{48 \pi^{2}} B^{2}-\frac{m}{6 \pi^{2}} \partial^{2} A_{0}+\frac{m^{3}}{\pi^{2}} A_{0}^{2}+\mathcal{O}\left(A_{\mu}^{3}\right) .
$$

The trace of the stress-energy tensor is finally given by

$$
\left\langle T_{i}^{i}-2 T_{0}^{0}\right\rangle=-\frac{1}{2} a_{4}\left(D_{E}^{2}\right)=\frac{m}{12 \pi^{2}} \partial^{2} A_{0}-\frac{m^{3}}{2 \pi^{2}} A_{0}^{2}+\frac{m}{96 \pi^{2}} B^{2}+\mathcal{O}\left(A_{\mu}^{3}\right) .
$$

The structure is the same as the bosonic case. 


\section{DIFFEOMORPHISM ANOMALY}

The previous calculation of the Weyl anomaly, which related the trace of the energy-momentum tensor with the $a_{4}$ coefficient, relies on the $\zeta$ function regularization. This method is described in [49] and here we sketch the derivation. ${ }^{7}$ For an operator $\mathcal{D}$ defining a classical action, the regularized vacuum functional is defined by

$W^{\mathrm{reg}}(s)=-\frac{1}{2} \tilde{\mu}^{2 s} \int_{0}^{\infty} \frac{d t}{t^{1-s}} \tilde{K}_{\mathcal{D}}(t)=-\frac{1}{2} \tilde{\mu}^{2 s} \Gamma(s) \zeta(s, \mathcal{D})$,

where the regulator is $s$, and $\tilde{\mu}$ is the mass parameter that any regularization procedure entails. The last equality gives the relation between the HK spectral function and the zeta function of an operator

$$
\zeta(s, \mathcal{D})=\frac{1}{\Gamma(s)} \int_{0}^{\infty} \frac{d t}{t^{1-s}} \tilde{K}_{\mathcal{D}}(t)=\operatorname{Tr}\left(\mathcal{D}^{-s}\right) .
$$

The physical limit of the regularized vacuum functional is attained for $s \rightarrow 0$ that, due to the presence of the $\Gamma$ function, develops an UV singularity that needs to be subtracted. The renormalized vacuum functional is the $s \rightarrow 0$ limit of the subtracted vacuum functional, leading to

$$
W^{\text {ren }}=-\frac{1}{2} \zeta^{\prime}(0, \mathcal{D})-\frac{1}{2} \log \mu^{2} \zeta(0, \mathcal{D}),
$$

where $\mu^{2}=e^{-\gamma_{E}} \tilde{\mu}^{2}$ is the renormalization scale and $\gamma_{E}$ the Euler Mascheroni constant.

Next, we need to see how the renormalized vacuum functional varies under a variation $\delta \mathcal{D}$. This is completely determined by the variation of the $\zeta(s, \mathcal{D})$ function,

$$
\delta \zeta(s, \mathcal{D})=-s \operatorname{Tr}\left((\delta \mathcal{D}) \mathcal{D}^{-s-1}\right) .
$$

To compute the diffeomorphism anomaly, we need the variation $\delta \mathcal{D}$ under diffeomorphisms. In the scalar case, after integration by parts, the imaginary-time action can be put in the form

$$
S_{E}=\int d^{3} x \sqrt{g} \phi^{\dagger} \mathcal{D} \phi
$$

with

$\mathcal{D} \phi=i m v^{\mu} D_{\mu} \phi+\frac{i m}{\sqrt{g}} D_{\mu}\left(\sqrt{g} v^{\mu} \phi\right)-\frac{1}{\sqrt{g}} D_{\mu}\left(\sqrt{g} h^{\mu \nu} D_{\nu} \phi\right)$.

\footnotetext{
${ }^{7}$ Reference [49] is very exhaustive but also pretty long. For the benefit of the reader, we recall that Sec. II B in Ref. [49] deals with the spectral functions relevant to this section, whereas the part relevant to the conformal anomaly can be found in Sec. VII A of Ref. [49].

${ }^{8}$ Note that we need to compute the variation for $s \neq 0$ and eventually perform the $s \rightarrow 0$ limit.
}

We will only consider the variation under diffeomorphisms of this operator specializing to a flat background with a nonvanishing gauge field. The scalar operator specialized to this background is

$$
\begin{aligned}
\mathcal{D}_{0}= & 2 i m \partial_{0}-\partial_{i}^{2}+2 m^{2} A_{0}+m^{2} A_{i} A_{i} \\
& +2 i m A_{i} \partial_{i}+i m\left(\partial_{i} A_{i}\right),
\end{aligned}
$$

and it transforms under diffeomorphisms as

$$
\begin{aligned}
\delta \mathcal{D}_{0}= & -2 i m\left(\partial_{0} \varepsilon^{\mu}\right) \partial_{\mu}+2\left(\partial_{i} \varepsilon^{\mu}\right) \partial_{i} \partial_{\mu} \\
& +\left(\partial_{i}^{2} \varepsilon^{\mu}\right) \partial_{\mu}+2 m^{2} \varepsilon^{\mu}\left(\partial_{\mu} A_{0}\right) \\
& +2 i m \varepsilon^{\mu}\left(\partial_{\mu} A_{i}\right) \partial_{i}-2 i m A_{i}\left(\partial_{i} \varepsilon^{\mu}\right) \partial_{\mu} \\
& +i m \varepsilon^{\mu}\left(\partial_{i} \partial_{\mu} A_{i}\right)+2 m^{2} A_{i} \varepsilon^{\mu}\left(\partial_{\mu} A_{i}\right) .
\end{aligned}
$$

Now comes an important point, which permits us to understand which terms in Eq. (4.8) do indeed contribute to the anomaly. The $\zeta$ function (4.2) is a trace and, as such, due to the cyclicity properties, is invariant under the similarity transformation

$$
\zeta(s, \tilde{\mathcal{D}})=\zeta(s, \mathcal{D}) \quad \text { if } \tilde{\mathcal{D}}=e^{\hat{O}} \mathcal{D} e^{-\hat{O}} .
$$

Strictly speaking, this means that $\mathcal{D}$ and $\tilde{\mathcal{D}}$ have the same functional determinant. If we consider the redefinition (4.9) with

$$
\hat{O}=\alpha \xi^{\mu} \partial_{\mu},
$$

with $\alpha$ a real coefficient and $\xi^{\mu}$ transforming under diffeomorphisms as $\delta \xi^{\mu}=\varepsilon^{\mu}$, we find that

$$
\begin{aligned}
\tilde{\mathcal{D}_{0}=} & e^{\alpha \xi^{\mu} \partial_{\mu}} \mathcal{D}_{0} e^{-\alpha \xi^{\nu}} \partial_{\nu}=\mathcal{D}_{0}-2 i m \alpha\left(\partial_{0} \xi^{\mu}\right) \partial_{\mu} \\
& +2 \alpha\left(\partial_{i} \xi^{\mu}\right) \partial_{i} \partial_{\mu}+\alpha\left(\partial_{i}^{2} \xi^{\mu}\right) \partial_{\mu}+2 m^{2} \alpha \xi^{\mu}\left(\partial_{\mu} A_{0}\right) \\
& +2 m^{2} \alpha A_{i} \xi^{\mu}\left(\partial_{\mu} A_{i}\right)+2 i m \alpha \xi^{\mu}\left(\partial_{\mu} A_{i}\right) \partial_{i} \\
& +i m \alpha \xi^{\mu}\left(\partial_{\mu} \partial_{i} A_{i}\right)-2 i m \alpha A_{i}\left(\partial_{i} \xi^{\mu}\right) \partial_{\mu}+\mathcal{O}\left(\xi^{2}\right) .
\end{aligned}
$$

Using Eq. (4.8) and setting $\alpha=-1$, we obtain $\delta \tilde{\mathcal{D}}_{0}=0$. This means that $\delta W^{\text {ren }}=0$, and there is no gravitational anomaly:

$$
\left\langle\partial_{\mu} T_{\nu}^{\mu}\right\rangle=0
$$

As a consequence, the divergence of the scale current in Eq. (2.18) takes the form $\left\langle\partial_{\mu} J_{S}^{\mu}\right\rangle=\left\langle T^{i}{ }_{i}-2 \epsilon^{0}\right\rangle$. On the contrary, in Ref. [19], a nonvanishing diffeomorphism anomaly was found using Fujikawa's method, i.e.,

$$
\left\langle\partial_{\mu}\left(T_{F}\right)^{\mu}{ }_{\nu}\right\rangle=\frac{1}{2} \partial_{\nu} \mathcal{A},
$$


where $\mathcal{A}$ is the trace anomaly evaluated from the energymomentum tensor $\left(T_{F}\right)^{\mu}{ }_{\nu}$, regularized as in Ref. [19]. This is not in contradiction with our result, as a "subtracted" energy-momentum tensor, in the spirit of Ref. [37], can be defined

$$
\hat{T}^{\mu}{ }_{\nu}=\left(T_{F}\right)^{\mu}{ }_{\nu}-\frac{1}{2} \delta^{\mu}{ }_{\nu} \mathcal{A},
$$

in such a way that it satisfies the conservation equation

$$
\left\langle\partial_{\mu} \hat{T}_{\nu}^{\mu}\right\rangle=0
$$

It seems that the zeta function regularization method we used automatically selects the conserved energy-momentum tensor $T^{\mu}{ }_{\nu}=\hat{T}^{\mu}{ }_{\nu}$. In fact, if we compare the trace of the subtracted $\hat{T}^{\mu}{ }_{\nu}$ with Eq. (3.27), we find a substantial agreement with $[19]^{9}$

\section{CONCLUSIONS}

In this paper we found that a nonzero trace anomaly occurs for a nonrelativistic scalar and fermion fields in $2+$ 1 dimensions, coupled to a particle number background $A_{\mu}$. This agrees with the results of [19]. Analogous calculations $[17,18]$ performed in curved backgrounds without $A_{\mu}$ lead to a result proportional to the trace anomaly of a relativistic scalar/fermion in $3+1$ dimensions. Instead, the anomaly in $A_{\mu}$ background is genuinely nonrelativistic, as it has no counterpart in the relativistic case. The resulting anomaly is not gauge invariant in the background source vector field; similar non-gauge-invariant anomalies are known to occur also in the relativistic case; see, e.g., [30,31].

We also computed the diffeomerphism anomaly for a scalar and we found a vanishing result; this may be not in contradiction with the results of [19], because our energymomentum tensor may correspond to a subtracted version of the one studied in [19].

Several open question are left for further investigation:

(i) An analysis of the Wess-Zumino consistency conditions for trace anomalies in presence of gauge and Milne-boost violations would clarify the nature of the anomalies and their possible relevance for the properties of the RG flow. Due to the large number of terms involved, this seems a rather challenging task.

(ii) It would be interesting to study nonrelativistic anomalies for supersymmetric theories. In the Schrödinger case it is likely that, in analogy to the relativistic case, a nontrivial relation between superconformal R-charge and trace anomaly exists at the fixed point $[33,50]$. In the relativistic case, the traditional derivation of these result relies on the equality of the flavor- $U(1)_{R^{-}} U(1)_{R}$ and flavor-gravity-gravity

\footnotetext{
${ }^{9}$ The disagreement concerns essentially an overall sign. We were not able to figure out what is the origin of the discrepancy.
}

triangle anomaly, due to supersymmetry. No axial anomaly is known in the Schrödinger case, ${ }^{10}$ and this makes the extension to the nonrelativistic case not straightforward. Another derivation of the relation between R-charges and $a$-anomaly was given in [32], using the local RG approach by Osborn [5]; the relevant Wess-Zumino consistency conditions concerned terms in the trace anomaly which are not formally gauge invariant [31]. It could be that a similar analysis might be extended to the nonrelativistic case.

(iii) An understanding of the case of anyons would be important for possible condensed matter applications.

\section{ACKNOWLEDGMENTS}

We are grateful to Arpita Mitra and Karan Fernandes for useful discussions.

\section{APPENDIX A: WEYL AND MILNE-BOOST TRANSFORMATIONS}

The Newton-Cartan fields transform under Milne boosts in the following way:

$v^{\prime \mu}=v^{\mu}+h^{\mu \nu} \psi_{\nu}$

$h_{\mu \nu}^{\prime}=h_{\mu \nu}-\left(n_{\mu} P_{\nu}^{\rho}+n_{\nu} P_{\mu}^{\rho}\right) \psi_{\rho}+n_{\mu} n_{\nu} h^{\rho \sigma} \psi_{\rho} \psi_{\sigma}$,

$A_{\mu}^{\prime}=A_{\mu}+P_{\mu}^{\rho} \psi_{\rho}-\frac{1}{2} n_{\mu} h^{\rho \sigma} \psi_{\rho} \psi_{\sigma}$,

where $\psi_{\mu}$ is the local parameter of the trasformations. The fields $n_{\mu}$ and $h^{\mu \nu}$ are invariant. These transformations are naturally implemented via null reduction technique.

The fundamental fields of Newton-Cartan geometry change as follow under 2+1-dimensional Weyl transformations:

$$
\begin{aligned}
n_{\mu} & \rightarrow e^{2 \sigma} n_{\mu}, & v^{\mu} & \rightarrow e^{-2 \sigma} v^{\mu}, \\
h_{\mu \nu} & \rightarrow e^{2 \sigma} h_{\mu \nu}, & h^{\mu \nu} & \rightarrow e^{-2 \sigma} h^{\mu \nu},
\end{aligned}
$$

where $\sigma$ is a local parameter and coordinates do not transform. Note that the gauge field $A_{\mu}$ is invariant.

The Newton-Cartan measure $\sqrt{g}$ changes under $2+1$ dimensional Weyl transormations as

$$
\sqrt{\operatorname{det}\left(h_{\mu \nu}+n_{\mu} n_{\nu}\right)}=\sqrt{g} \rightarrow e^{4 \sigma} \sqrt{g}
$$

\section{APPENDIX B: CONVENTIONS ON GAMMA MATRICES AND VIELBEIN}

The $2+1$-dimensional dreibein $e_{\mu}^{a}$ is defined by dimensional reduction of the $3+1$-dimensional fierbein $e_{M}^{A}$ :

\footnotetext{
${ }^{10}$ Axial anomaly instead was already studied in the Lifshitz case; see [51].
} 
$e_{M}^{A}=\left(\begin{array}{c}e^{-}{ }_{M} \\ e^{+}{ }_{M} \\ e^{a}{ }_{M}\end{array}\right)=\left(\begin{array}{ll}e^{-}- & e^{-}{ }_{\mu} \\ e^{+}- & e^{+}{ }_{\mu} \\ e^{a}- & e^{a}{ }_{\mu}\end{array}\right)=\left(\begin{array}{cc}1 & A_{\mu} \\ 0 & n_{\mu} \\ \mathbf{0} & e_{\mu}^{a}\end{array}\right)$.

The inverse vielbein are

$$
\begin{aligned}
e_{A}^{M_{A}} & =\left(\begin{array}{ccc}
e^{M}- & e^{M}+ & e^{M}{ }_{a}
\end{array}\right)=\left(\begin{array}{ccc}
e^{-} & e^{-} & e^{-}{ }_{a} \\
e^{\mu} & e^{\mu} & e^{\mu}{ }_{a}
\end{array}\right) \\
& =\left(\begin{array}{ccc}
1 & -v^{\sigma} A_{\sigma} & -h^{\nu \sigma} A_{\sigma} e_{\nu}^{a} \\
\mathbf{0} & v^{\mu} & h^{\mu \nu} e_{\nu}^{a}
\end{array}\right) .
\end{aligned}
$$

The $2+1$-dimensional dreibein $e^{a}{ }_{\mu}$ are not completely free, but they are related to other Newton-Cartan fields via the relations

$$
e^{M}{ }_{A} e^{B}{ }_{M}=\delta_{A}^{B}, \quad e^{A}{ }_{M} e^{N}{ }_{A}=\delta_{M}{ }^{N} .
$$

In order to deal with nonrelativistic fermions using the null-reduction method, it is necessary to introduce Pauli and Dirac matrices in four dimensions in light-cone indices. The usual convention is

$$
\sigma^{A}=\left(\mathbf{1}, \sigma^{\alpha}\right), \quad \bar{\sigma}^{A}=\left(-\mathbf{1}, \sigma^{\alpha}\right)
$$

In light-cone coordinates, they become

$$
\begin{array}{ll}
\sigma^{ \pm}=\frac{1}{\sqrt{2}}\left(\sigma^{3} \pm \sigma^{0}\right), & \bar{\sigma}^{ \pm}=\frac{1}{\sqrt{2}}\left(\bar{\sigma}^{3} \pm \bar{\sigma}^{0}\right), \\
\sigma^{-}=\sqrt{2}\left(\begin{array}{cc}
0 & 0 \\
0 & -1
\end{array}\right), & \sigma^{+}=\sqrt{2}\left(\begin{array}{cc}
1 & 0 \\
0 & 0
\end{array}\right), \\
\bar{\sigma}^{-}=\sqrt{2}\left(\begin{array}{cc}
1 & 0 \\
0 & 0
\end{array}\right), & \bar{\sigma}^{+}=\sqrt{2}\left(\begin{array}{cc}
0 & 0 \\
0 & -1
\end{array}\right), \\
\sigma^{1}=\bar{\sigma}^{1}=\left(\begin{array}{ll}
0 & 1 \\
1 & 0
\end{array}\right), & \sigma^{2}=\bar{\sigma}^{2}=\left(\begin{array}{cc}
0 & -i \\
i & 0
\end{array}\right) .
\end{array}
$$

The associated Gamma matrices in four dimensions are

$$
\begin{aligned}
& \gamma^{-}=\frac{1}{\sqrt{2}}\left(\gamma^{3}-\gamma^{0}\right)=\sqrt{2}\left(\begin{array}{cccc}
0 & 0 & 0 & 0 \\
0 & 0 & 0 & -1 \\
1 & 0 & 0 & 0 \\
0 & 0 & 0 & 0
\end{array}\right), \quad \gamma^{+}=\frac{1}{\sqrt{2}}\left(\gamma^{3}+\gamma^{0}\right)=\sqrt{2}\left(\begin{array}{cccc}
0 & 0 & 1 & 0 \\
0 & 0 & 0 & 0 \\
0 & 0 & 0 & 0 \\
0 & -1 & 0 & 0
\end{array}\right), \\
& \gamma^{1}=\left(\begin{array}{cc}
0 & \sigma^{1} \\
\sigma^{1} & 0
\end{array}\right), \quad \gamma^{2}=\left(\begin{array}{cc}
0 & \sigma^{2} \\
\sigma^{2} & 0
\end{array}\right) .
\end{aligned}
$$

The corresponding Lorentz generators, needed to compute covariant derivatives, are

$$
\sigma^{A B}=\frac{1}{2}\left(\sigma^{A} \bar{\sigma}^{B}-\sigma^{B} \bar{\sigma}^{A}\right), \quad \gamma^{A B}=\frac{1}{2}\left[\gamma^{A}, \gamma^{B}\right]
$$

\section{APPENDIX C: TIME-INDEPENDENT INSERTION CONTRIBUTIONS TO HEAT KERNEL}

In this Appendix, we will compute time-independent insertion of operators in the heat kernel expansion. We derive the formula for a single insertion of the term $Q_{i}(x)$ multiplying a spatial derivative; all the other nonvanishing insertions can be found in [17-18]. We compute

$$
\begin{aligned}
K_{1 Q_{i}}(s) & =\int_{0}^{s^{\prime}} d s^{\prime} \int d^{d} \tilde{x} \int d \tilde{t}\left\langle x t\left|e^{\left(s-s^{\prime}\right) \triangle}\right| \tilde{x} \tilde{t}\right\rangle Q_{i}(\tilde{x}) \frac{\partial}{\partial \tilde{x}_{i}}\left\langle\tilde{x} \tilde{t}\left|e^{s^{\prime} \triangle}\right| x^{\prime} t^{\prime}\right\rangle= \\
& =-\frac{\partial}{\partial x_{i}^{\prime}}\left[\int_{0}^{s^{\prime}} d s^{\prime} \int d^{d} \tilde{x} \int d \tilde{t}\left\langle x t\left|e^{\left(s-s^{\prime}\right) \triangle}\right| \tilde{x} \tilde{t}\right\rangle Q_{i}(\tilde{x})\left\langle\tilde{x} \tilde{t}\left|e^{s^{\prime} \triangle}\right| x^{\prime} t^{\prime}\right\rangle\right],
\end{aligned}
$$

where we used the parity properties of the flat solution of the heat kernel and of the derivative operation. In this way we recognize that the term in parenthesis is the definition of the single insertion of a term without derivatives of kind

$$
K_{1 P}(s)=\int_{0}^{s^{\prime}} d s^{\prime} \int d^{d} \tilde{x} \int d \tilde{t}\left\langle x t\left|e^{\left(s-s^{\prime}\right) \Delta}\right| \tilde{x} \tilde{t}\right\rangle P(\tilde{x})\left\langle\tilde{x} \tilde{t}\left|e^{s^{\prime} \triangle}\right| x^{\prime} t^{\prime}\right\rangle
$$

where we only have to rename the operator as $Q_{i}(x)$. 
The computation for this quantity was performed in [17] and gave as a result

$$
\begin{aligned}
K_{1 P}(s)= & \int_{0}^{s} d s^{\prime} \frac{1}{2 \pi^{2}} \frac{1}{(4 \pi s)^{d / 2}} \frac{8 \pi m s}{4 m^{2} s^{2}+\left(t-t^{\prime}\right)^{2}} \\
& \times \int \frac{d^{d} k}{(2 \pi)^{d / 2}} \exp \left(-\frac{\left(x-x^{\prime}\right)^{2}}{4 s}+i k \cdot\left(x \frac{s^{\prime}}{s}+x^{\prime} \frac{s-s^{\prime}}{s}\right)-k^{2} \frac{s^{\prime}}{s}\left(s-s^{\prime}\right)\right) Q_{i}(k) .
\end{aligned}
$$

If we now differentiate this expression with respect to $x^{\prime}$ and we put $t=t^{\prime}, x=x^{\prime}$, we obtain

$$
\tilde{K}_{1 Q_{i}}(s)=\int_{0}^{s} d s^{\prime} \int \frac{d^{d} k}{(2 \pi)^{d / 2}} \frac{2}{m(4 \pi s)^{d / 2+1}}\left(-i k_{i} \frac{s-s^{\prime}}{s}\right) \exp \left(i k x-k^{2} \frac{s^{\prime}}{s}\left(s-s^{\prime}\right)\right) Q_{i}(k) .
$$

Performing the inverse Fourier transform and expanding the exponential around $s=0$, we find the result

$$
\tilde{K}_{1 Q_{i}}(s)=\frac{2}{m(4 \pi s)^{d / 2+1}}\left(-\frac{s}{2} \partial_{i} Q_{i}(x)-\frac{s^{2}}{12} \partial_{i} \partial^{2} Q_{i}(x)+\mathcal{O}\left(s^{3}\right)\right) .
$$

\section{APPENDIX D: TIME-DEPENDENT INSERTION CONTRIBUTIONS TO HEAT KERNEL}

We want to generalize the results of the previous section concerning the heat kernel expansion by considering timedependent insertions of operators.

\section{Single-insertion computations}

Let us start with the single insertion of a term without derivatives acting on the fields. We consider the Fourier decomposition,

$$
P(x, t)=\int \frac{d^{d} k}{(2 \pi)^{d / 2}} \int \frac{d \omega}{\sqrt{2 \pi}} P(k, \omega) e^{i(k x-\omega t)},
$$

in order to find

$$
\begin{aligned}
K_{1 P}(s)= & \int_{0}^{s} d s^{\prime} \int d^{d} \tilde{x} \int d \tilde{t}\left\langle x t\left|e^{\left(s-s^{\prime}\right) \Delta}\right| \tilde{x} \tilde{t}\right\rangle P(\tilde{x}, \tilde{t})\left\langle\tilde{x} \tilde{t}\left|e^{s^{\prime} \Delta}\right| x^{\prime} t^{\prime}\right\rangle \\
= & \int_{0}^{s} d s^{\prime} \frac{1}{(2 \pi)^{2}} \frac{1}{\left(4 \pi\left(s-s^{\prime}\right)\right)^{d / 2}} \frac{1}{\left(4 \pi s^{\prime}\right)^{d / 2}} \int \frac{d \omega}{\sqrt{2 \pi}} \int d \tilde{t} e^{-i \omega \tilde{t}} \frac{m\left(s-s^{\prime}\right)}{m^{2}\left(s-s^{\prime}\right)^{2}+\frac{(t-\tilde{t})^{2}}{4}} \\
& \times \frac{m s^{\prime}}{m^{2} s^{\prime 2}+\frac{\left(\tilde{t}-t^{\prime}\right)^{2}}{4}} \int \frac{d^{d} k}{(2 \pi)^{d / 2}} e^{i k \tilde{x}} \exp \left(-\frac{(x-\tilde{x})^{2}}{4\left(s-s^{\prime}\right)}-\frac{\left(\tilde{x}-x^{\prime}\right)^{2}}{4 s^{\prime}}\right) P(k, \omega),
\end{aligned}
$$

where we used the explicit expression of the flat-space heat kernel for the Schrödinger operator, Eq. (3.8).

We observe that the time and spatial parts of the integral decouple and appear as distinct multiplicative factors. We can then use the result for the spatial part that is presented in [17]:

$$
\begin{aligned}
& \int d^{d} \tilde{x} \int \frac{d^{d} k}{(2 \pi)^{d / 2}} e^{i k \tilde{x}} \exp \left(-\frac{(x-\tilde{x})^{2}}{4\left(s-s^{\prime}\right)}-\frac{\left(\tilde{x}-x^{\prime}\right)^{2}}{4 s^{\prime}}\right) \\
& =\int \frac{d^{d} k}{2 \pi^{d / 2}} \exp \left(-\frac{\left(x-x^{\prime}\right)^{2}}{4 s}+i k \cdot\left(x \frac{s^{\prime}}{s}+x^{\prime} \frac{s-s^{\prime}}{s}\right)-k^{2} \frac{s^{\prime}}{s}\left(s-s^{\prime}\right)\right) .
\end{aligned}
$$

In particular, the $x=x^{\prime}$ result for the spatial part of the integral is

$$
\int \frac{d^{d} k}{2 \pi^{d / 2}} \exp \left(i k \cdot x-k^{2} \frac{s^{\prime}}{s}\left(s-s^{\prime}\right)\right)
$$


In order to compute the temporal part $I(\omega)$ of the integral, we need to find the analytic structure in the complex plane of the integrand,

$$
\begin{aligned}
I(\omega) & =\int d \tilde{t} e^{-i \omega \tilde{t}} \frac{m\left(s-s^{\prime}\right)}{m^{2}\left(s-s^{\prime}\right)^{2}+\frac{(t-\tilde{t})^{2}}{4}} \frac{m s^{\prime}}{m^{2} s^{\prime 2}+\frac{\left(\tilde{t}-t^{\prime}\right)^{2}}{4}} \\
& =4 \alpha \beta e^{-i \omega t^{\prime}} \int d \tilde{t} \frac{e^{-i \omega \tilde{t}}}{(\tilde{t}+i \beta)(\tilde{t}-i \beta)(\tilde{t}-\Delta t+i \alpha)(\tilde{t}-\Delta t-i \alpha)},
\end{aligned}
$$

where we sent $\tilde{t} \rightarrow \tilde{t}+t^{\prime}$ and we defined

$$
\alpha=2 m\left(s-s^{\prime}\right), \quad \beta=2 m s^{\prime}, \quad \Delta t=t-t^{\prime} .
$$

The quantities $\alpha, \beta$ are positive by definition. We use the residue theorem to find

$$
\begin{aligned}
I(\omega)= & 4 \alpha \beta e^{-i \omega t^{\prime}} \theta(\omega)\left[\frac{\pi e^{-\beta \omega}}{\beta\left((\Delta t+i \beta)^{2}+\alpha^{2}\right)}+\frac{\pi e^{-\alpha \omega-i \Delta t \omega}}{\alpha\left((\Delta t-i \alpha)^{2}+\beta^{2}\right)}\right] \\
& +4 \alpha \beta e^{-i \omega t^{\prime}} \theta(-\omega)\left[\frac{\pi e^{\beta \omega}}{\beta\left((\Delta t-i \beta)^{2}+\alpha^{2}\right)}+\frac{\pi e^{\alpha \omega-i \Delta t \omega}}{\alpha\left((\Delta t+i \alpha)^{2}+\beta^{2}\right)}\right] .
\end{aligned}
$$

It can be found that the expression for $\omega=0$ gives the time-independent results found in [17-18] if we choose the prescription $\theta(0)=1 / 2$ for the Heaviside distribution:

$$
I(0)=\frac{8 \pi m s}{4 m^{2} s^{2}+\left(t-t^{\prime}\right)^{2}}=\int d \tilde{t} \frac{m\left(s-s^{\prime}\right)}{m^{2}\left(s-s^{\prime}\right)^{2}+\frac{(t-\tilde{t})^{2}}{4}} \frac{m s^{\prime}}{m^{2} s^{\prime 2}+\frac{\left(\tilde{t}-t^{\prime}\right)^{2}}{4}} .
$$

The heat kernel computation only requires equal-time insertions, then we put $\Delta t=0$ to obtain

$$
I(\omega, \Delta t=0)=\frac{2 \pi}{m s} \frac{1}{s-2 s^{\prime}}\left[e^{-2 m s^{\prime}|\omega|}\left(s-s^{\prime}\right)-s^{\prime} e^{-2 m\left(s-s^{\prime}\right)|\omega|}\right]=\frac{2 \pi}{m s}+\mathcal{O}(s) .
$$

Using Eqs. (D4) and (D9) inside Eq. (D2) and expanding in the auxiliary time $s$, we finally obtain the result:

$$
\tilde{K}_{1 P}(s)=\frac{2}{m(4 \pi s)^{d / 2+1}}\left(s P(x, t)+\frac{1}{6} s^{2} \partial_{i}^{2} P(x, t)+\mathcal{O}\left(s^{3}\right)\right) .
$$

This is the same result as the case without time dependence because the first order of the expansion of exponential terms vanishes.

Let us now consider the single insertion of an operator with a spatial derivative acting on the fields. Once again, we find that Eq. (C2) is satisfied and then the calculation reduces to applying a spatial derivative to the single insertion $K_{1 P}(s)$ :

$$
K_{1 Q_{i}}(s)=-\frac{\partial}{\partial x_{i}^{\prime}}\left[\int_{0}^{s^{\prime}} d s^{\prime} \int d^{d} \tilde{x} \int d \tilde{t}\left\langle x t\left|e^{\left(s-s^{\prime}\right) \Delta}\right| \tilde{x} \tilde{t}\right\rangle Q_{i}(\tilde{x}, \tilde{t})\left\langle\tilde{x} \tilde{t}\left|e^{s^{\prime} \Delta}\right| x^{\prime} t^{\prime}\right\rangle\right] .
$$

Since the expression in parentheses does not change if we add a time dependence to the operators of the heat-kernel expansion, and since spatial and temporal parts of the integral factorize, we obtain an equivalent formula also for

$$
\tilde{K}_{1 Q_{i}}(s)=\frac{2}{m(4 \pi s)^{d / 2+1}}\left(-\frac{s}{2} \partial_{i} Q_{i}(x, t)-\frac{s^{2}}{12} \partial_{i} \partial^{2} Q_{i}(x, t)+\mathcal{O}\left(s^{3}\right)\right) .
$$

Single insertions of operators with a time derivative applied to the dynamical fields $S(x, t)$ can be modified by time dependence, but since they vanish on our background, we will not consider this kind of term. 


\section{Double-insertion computations}

We consider the double insertion of operators of type $P(x, t)$, which is given by

$$
\begin{aligned}
K_{2 P}(s)= & \int_{0}^{s} d s_{2} \int_{0}^{s_{2}} d s_{1} \int d^{d} x_{1} \int d^{d} x_{2} \int d t_{1} \int d t_{2}\left\langle x t\left|e^{\left(s-s_{2}\right) \Delta}\right| x_{2} t_{2}\right\rangle \\
P & \left(x_{2}, t_{2}\right)\left\langle x_{2} t_{2}\left|e^{\left(s_{2}-s_{1}\right) \Delta}\right| x_{1} t_{1}\right\rangle P\left(x_{1}, t_{1}\right)\left\langle x_{1} t_{1}\left|e^{s_{1} \Delta}\right| x^{\prime} t^{\prime}\right\rangle \\
= & \int_{0}^{s} d s_{2} \int_{0}^{s_{2}} d s_{1} \frac{1}{(2 \pi)^{3}} \frac{1}{\left(4 \pi\left(s-s_{2}\right)\right)^{d / 2}} \frac{1}{\left(4 \pi\left(s_{2}-s_{1}\right)\right)^{d / 2}} \frac{1}{\left(4 \pi s_{1}\right)^{d / 2}} \int d^{d} x_{1} \int d^{d} x_{2} \int d t_{1} \int d t_{2} \\
& \times \int \frac{d^{d} k_{1}}{(2 \pi)^{d / 2}} \int \frac{d^{d} k_{2}}{(2 \pi)^{d / 2}} \exp \left(i k_{1} x_{1}+i k_{2} x_{2}-\frac{\left(x^{\prime}-x_{2}\right)^{2}}{4\left(s-s_{2}\right)}-\frac{\left(x_{2}-x_{1}\right)^{2}}{4\left(s_{2}-s_{1}\right)}-\frac{\left(x_{1}-x\right)^{2}}{4 s_{1}}\right) \int \frac{d \omega_{1}}{\sqrt{2 \pi}} \int \frac{d \omega_{2}}{\sqrt{2 \pi}} \\
& \times e^{-i \omega_{1} t_{1}-i \omega_{2} t_{2}} \frac{m\left(s-s_{2}\right)}{m^{2}\left(s-s_{2}\right)^{2}+\frac{\left(t-t_{2}\right)^{2}}{4}} \frac{m\left(s_{2}-s_{1}\right)}{m^{2}\left(s_{2}-s_{1}\right)^{2}+\frac{\left(t_{2}-t_{1}\right)^{2}}{4}} \frac{m s_{1}}{m^{2} s_{1}^{2}+\frac{\left(t_{1}-t^{\prime}\right)^{2}}{4}} P\left(k_{2}, \omega_{2}\right) P\left(k_{1}, \omega_{1}\right) .
\end{aligned}
$$

It is evident that also in this situation the time and spatial parts of the integral factorize. The latter was found in [17] to be

$$
\begin{aligned}
\Xi\left(x, x^{\prime}\right)= & \int d^{d} x_{1} \int d^{d} x_{2} \exp \left(-\frac{\left(x^{\prime}-x_{2}\right)^{2}}{4\left(s-s_{2}\right)}-\frac{\left(x_{2}-x_{1}\right)^{2}}{4\left(s_{2}-s_{1}\right)}-\frac{\left(x_{1}-x\right)^{2}}{4 s_{1}}+i k_{1} x_{1}+i k_{2} x_{2}\right) \\
= & (4 \pi)^{d}\left(\frac{s_{1}\left(s-s_{2}\right)\left(s_{2}-s_{1}\right)}{s}\right)^{d / 2} \exp \left(\frac{i k_{1} s_{1} x^{\prime}}{s}+\frac{i k_{2} s_{2} x^{\prime}}{s}-\frac{i k_{1} s_{1} x}{s}-\frac{i k_{2} s_{2} x}{s}+\frac{k_{1}^{2} s_{1}^{2}}{s}+\frac{k_{2}^{2} s_{2}^{2}}{s}\right. \\
& \left.-k_{1}^{2} s_{1}-2 k_{1} k_{2} s_{1}-k_{2}^{2} s_{2}+\frac{2 k_{1} k_{2} s_{1} s_{2}}{s}+i k_{1} x+i k_{2} x-\frac{x^{2}}{4 s}+\frac{x x^{\prime}}{2 s}-\frac{\left(x^{\prime}\right)^{2}}{4 s}\right) .
\end{aligned}
$$

At coincident points, it becomes

$$
\begin{aligned}
\Xi(x, x)= & (4 \pi)^{d}\left(\frac{s_{1}\left(s-s_{2}\right)\left(s_{2}-s_{1}\right)}{s}\right)^{d / 2} \\
& \times \exp \left(i k_{1} x_{1}+i k_{2} x_{2}+k_{1}^{2}\left(\frac{s_{1}^{2}}{s}-s_{1}\right)+k_{2}^{2}\left(\frac{s_{2}^{2}}{s}-s_{2}\right)+2 k_{1} k_{2}\left(\frac{s_{1} s_{2}}{s}-s_{1}\right)\right) .
\end{aligned}
$$

Now we analyze the temporal part:

$$
\Psi\left(t, t^{\prime}, \omega_{1}, \omega_{2}\right)=\int d t_{1} \int d t_{2} e^{-i \omega_{1} t_{1}-i \omega_{2} t_{2}} \frac{m\left(s-s_{2}\right)}{m^{2}\left(s-s_{2}\right)^{2}+\frac{\left(t-t_{2}\right)^{2}}{4}} \frac{m\left(s_{2}-s_{1}\right)}{m^{2}\left(s_{2}-s_{1}\right)^{2}+\frac{\left(t_{2}-t_{1}\right)^{2}}{4}} \frac{m s_{1}}{m^{2} s_{1}^{2}+\frac{\left(t_{1}-t^{\prime}\right)^{2}}{4}} .
$$

The integral in the variable $t_{1}$ can be performed using the previous technique. If we set

$$
\alpha=2 m\left(s_{2}-s_{1}\right), \quad \beta=2 m s_{1}, \quad \Delta t=t_{2}-t^{\prime},
$$

we find

$$
\begin{aligned}
I\left(\omega_{1}\right)= & \int d t_{1} e^{-i \omega_{1} t_{1}} \frac{m\left(s_{2}-s_{1}\right)}{m^{2}\left(s_{2}-s_{1}\right)^{2}+\frac{(t-\tilde{t})^{2}}{4} \frac{m s_{1}}{m^{2} s_{1}^{2}+\frac{(t-\tilde{t})^{2}}{4}}} \\
= & 4 \alpha \beta e^{-i \omega t^{\prime}} \theta\left(\omega_{1}\right)\left[\frac{\pi e^{-\beta \omega_{1}}}{\beta\left((\Delta t+i \beta)^{2}+\alpha^{2}\right)}+\frac{\pi e^{-\alpha \omega-i \Delta t \omega}}{\alpha\left((\Delta t-i \alpha)^{2}+\beta^{2}\right)}\right] \\
& +4 \alpha \beta e^{-i \omega t^{\prime}} \theta\left(-\omega_{1}\right)\left[\frac{\pi e^{\beta \omega}}{\beta\left((\Delta t-i \beta)^{2}+\alpha^{2}\right)}+\frac{\pi e^{\alpha \omega-i \Delta t \omega}}{\alpha\left((\Delta t+i \alpha)^{2}+\beta^{2}\right)}\right] .
\end{aligned}
$$

The last step in the time integration consists in evaluating 
$\Psi\left(t, t^{\prime}, \omega_{1}, \omega_{2}\right)=\int d t_{2} e^{-i \omega_{2} t_{2}} \frac{m\left(s-s_{2}\right)}{m^{2}\left(s-s_{2}\right)^{2}+\frac{\left(t-t_{2}\right)^{2}}{4}} I\left(\omega_{1}\right)$.

The result of the evaluation is very cumbersome, but it can be checked that, assuming the prescription $\theta(0)=1 / 2$, it gives the exact time-independent result in the limit of vanishing frequencies:

$$
\Psi\left(t=t^{\prime}, \omega_{1}=\omega_{2}=0\right)=\frac{16 \pi^{2} \theta^{2}(0)}{m s}=\frac{4 \pi^{2}}{m s} .
$$

Moreover, in order to compute the insertions of timedependent operators, we only need the lowest orders of the expansion in $s$ of the solution at coincident points, which turns out to be

$$
\Psi\left(t=t^{\prime}, \omega_{1}, \omega_{2}\right)=\frac{4 \pi^{2}}{m s} e^{-i\left(\omega_{1}+\omega_{2}\right) t}+\mathcal{O}(s) .
$$

The zeroth order in the variable $s$ vanishes.
Combining Eqs. (D14) and (D20) into (D13), we find the same result as the time-independent case:

$$
\tilde{K}_{2 P P}=\frac{2}{m(4 \pi s)^{d / 2+1}}\left(\frac{s^{2}}{2} P(x, t)^{2}+\mathcal{O}\left(s^{3}\right)\right) .
$$

Additional new terms will contribute only to higher orders in $s$, and therefore they do not modify the $a_{4}$ coefficient.

Since time and space integrals factorize and there are no contributions to lower-order terms in the heat-kernel expansion, we can similarly find that $\tilde{K}_{2 X}$ have the same expressions of the time-independent case, if we choose among the set

$$
X=\left\{P(x, t), Q_{i}(x, t)\right\}
$$

Additional terms could appear in insertions concerning the operator $S(x, t)$. They will not be considered here because $S(x, t)$ vanishes in all the backgrounds studied in this paper.
[1] A. B. Zamolodchikov, Pis'ma Zh. Eksp. Teor. Fiz. 43, 565 (1986) [JETP Lett. 43, 730 (1986)].

[2] J. L. Cardy, Phys. Lett. B 215, 749 (1988).

[3] H. Osborn, Phys. Lett. B 222, 97 (1989).

[4] I. Jack and H. Osborn, Nucl. Phys. B343, 647 (1990).

[5] H. Osborn, Nucl. Phys. B363, 486 (1991).

[6] Z. Komargodski and A. Schwimmer, J. High Energy Phys. 12 (2011) 099.

[7] Z. Komargodski, J. High Energy Phys. 07 (2012) 069.

[8] D. T. Son and M. Wingate, Ann. Phys. (Amsterdam) 321, 197 (2006).

[9] C. Hoyos and D. T. Son, Phys. Rev. Lett. 108, 066805 (2012).

[10] D. T. Son, arXiv:1306.0638.

[11] M. Geracie, D. T. Son, C. Wu, and S. F. Wu, Phys. Rev. D 91, 045030 (2015).

[12] S. Deser and A. Schwimmer, Phys. Lett. B 309, 279 (1993).

[13] K. Jensen, arXiv:1412.7750.

[14] R. Auzzi, S. Baiguera, and G. Nardelli, J. High Energy Phys. 02 (2016) 003; 02, (2016) 177(E).

[15] I. Arav, S. Chapman, and Y. Oz, J. High Energy Phys. 06 (2016) 158.

[16] R. Auzzi, S. Baiguera, F. Filippini, and G. Nardelli, J. High Energy Phys. 11 (2016) 163.

[17] R. Auzzi and G. Nardelli, J. High Energy Phys. 07 (2016) 047.

[18] R. Auzzi, S. Baiguera, and G. Nardelli, J. High Energy Phys. 08 (2017) 042.

[19] K. Fernandes and A. Mitra, Phys. Rev. D 96, 085003 (2017).
[20] S. Pal and B. Grinstein, Phys. Rev. D 96, 125001 (2017).

[21] I. Adam, I. V. Melnikov, and S. Theisen, J. High Energy Phys. 09 (2009) 130.

[22] M. Baggio, J. de Boer, and K. Holsheimer, J. High Energy Phys. 07 (2012) 099.

[23] T. Griffin, P. Horava, and C. M. Melby-Thompson, J. High Energy Phys. 05 (2012) 010.

[24] I. Arav, S. Chapman, and Y. Oz, J. High Energy Phys. 02 (2015) 078.

[25] A. O. Barvinsky, D. Blas, M. Herrero-Valea, D. V. Nesterov, G. Prez-Nadal, and C. F. Steinwachs, J. High Energy Phys. 06 (2017) 063.

[26] I. Arav, Y. Oz, and A. Raviv-Moshe, J. High Energy Phys. 03 (2017) 088.

[27] S. Pal and B. Grinstein, J. High Energy Phys. 12 (2016) 012.

[28] S. L. Adler, Phys. Rev. 177, 2426 (1969).

[29] J. S. Bell and R. Jackiw, Nuovo Cimento A 60, 47 (1969).

[30] W. A. Bardeen, Phys. Rev. 184, 1848 (1969).

[31] B. Keren-Zur, J. High Energy Phys. 09 (2014) 011.

[32] R. Auzzi and B. Keren-Zur, J. High Energy Phys. 05 (2015) 150.

[33] K. A. Intriligator and B. Wecht, Nucl. Phys. B667, 183 (2003).

[34] M. Stone, Phys. Rev. B 85, 184503 (2012).

[35] A. Gromov and A. G. Abanov, Phys. Rev. Lett. 113, 266802 (2014).

[36] T. Can, M. Laskin, and P. Wiegmann, Ann. Phys. (Amsterdam) 362, 752 (2015).

[37] W. A. Bardeen and B. Zumino, Nucl. Phys. B244, 421 (1984). 
[38] C. Duval, G. Burdet, H. P. Kunzle, and M. Perrin, Phys. Rev. D 31, 1841 (1985).

[39] K. Jensen, arXiv:1408.6855.

[40] J. Hartong, E. Kiritsis, and N. A. Obers, Phys. Rev. D 92 , 066003 (2015).

[41] J. Hartong, E. Kiritsis, and N. A. Obers, Phys. Lett. B 746, 318 (2015).

[42] J. Hartong, E. Kiritsis, and N. A. Obers, J. High Energy Phys. 08 (2015) 006.

[43] M. Geracie, arXiv:1611.01198.

[44] R. Banerjee, A. Mitra, and P. Mukherjee, Classical Quantum Gravity 32, 045010 (2015).
[45] R. Banerjee and P. Mukherjee, Phys. Rev. D 93, 085020 (2016).

[46] R. Banerjee and P. Mukherjee, Classical Quantum Gravity 33, 225013 (2016).

[47] S. N. Solodukhin, J. High Energy Phys. 04 (2010) 101.

[48] S. M. Christensen and M. J. Duff, Nucl. Phys. B154, 301 (1979).

[49] D. V. Vassilevich, Phys. Rep. 388, 279 (2003).

[50] D. Anselmi, D. Z. Freedman, M. T. Grisaru, and A. A. Johansen, Nucl. Phys. B526, 543 (1998).

[51] I. Bakas and D. Lust, Fortschr. Phys. 59, 937 (2011). 